\title{
Contributos da literacia da informação para a Ciência Aberta
}

\author{
La contribución de la alfabetización informacional a la Ciencia Abierta
}

The contribution of information literacy to Open Science

\section{Carlos Lopes (1), Maria da Luz Antunes (2) Tatiana SAnches (3)}

(1) ISPA - Instituto Universitário de Ciências Psicológicas, Sociais e da Vida, R. Jardim do Tabaco, 34, 1149-041 Lisboa - Portugal, clopes@ispa.pt (2) Escola Superior de Tecnologia da Saúde de Lisboa, Instituto Politécnico de Lisboa, Av. D. João II, lote 4.69.01, 1990-096 Lisboa - Portugal, mluz.antunes@estesl.ipl.pt (3) Instituto de Educação, Universidade de Lisboa, Alameda da Universidade, 1649-013 Lisboa, e-mail: tsanches@fpie.ulisboa.pt. Los autores integran el APPsyCl - Applied Psychology Research Center Capabilities \& Inclusion (ISPA-Instituto Universitário).

\section{Resumen}

El campo de actuación de la Ciencia Abierta se traduce en un proceso colaborativo, transparente, de diseminación, creación y transferencia de conocimiento, accesible a la investigación y basado en los principios del acceso abierto. Los diferentes agentes del proceso de investigación, dotados de un conjunto de competencias de alfabetización de la información, adquieren la aptitud —en ambientes de información impresa o digital, y partiendo de su propio pensamiento crítico y reflexivo- de transformar la información en nuevo conocimiento. Este artículo explora la integración de los conceptos de la Ciencia Abierta en la alfabetización de la información. Se presenta una reflexión teórica que evidencia las contribuciones de la alfabetización de la información en el contexto académico y en la dinámica de la producción de ciencia. Se concluye que la alfabetización de la información se asume como una herramienta de aprendizaje esencial para el desarrollo de la Ciencia Abierta, potenciando el entendimiento crítico de los contenidos, junto con el desarrollo y el progreso de la investigación.

Palabras clave: Alfabetización informacional. Ciencia abierta. Acceso abierto. Investigación. Aprendizaje.

It is far better to foresee even without certainty than not to fore-see at all. (Jules Henri Poincaré, The foundations of science, 1913)

\section{Introdução}

As mudanças de paradigma comunicacional produziram consequências de nível social, político, económico e cultural, sendo que o paradigma do aparecimento da Internet e da digitalização progressiva da sociedade é aquele que requer maior exigência formativa, na medida em que os seus efeitos se multiplicam por todas as atividades, desde as estritamente pessoais às profissionais. Se esta é uma premissa válida para a sociedade em geral ainda mais o é em contexto académico, em que as transformações experimentadas nos últimos anos afetaram profundamente todo $\mathrm{o}$

\section{Abstract}

The scope of Open Science translates into a collaborative, transparent process of dissemination, creation, and transfer of knowledge, accessible to research and based on the principles of open access. The different agents of the research process, equipped with a set of information literacy skills, acquire the ability, in print or digital information environments, and based on their own critical and reflexive thinking, to transform information into new knowledge. This article explores the integration of Open Science concepts in information literacy. It presents a theoretical reflection that shows the contributions of information literacy in the academic context and in the dynamics of the production of science. It is concluded that information literacy is an essential learning tool for the development of Open Science, enhancing the critical understanding of content, as well as the development and progress of research.

Keywords: Information literacy. Open Science. Open access. Research. Learning.

coletivo, alterando a metodologia docente, os modelos de ensino, os sistemas de comunicação científica, etc., dando lugar a um novo paradigma educacional em que o digital e o tecnológico constituem a sua inspiração mais importante (Córdon, 2016). O novo paradigma tem feito o seu caminho na teoria e na prática. Neste contexto emerge o conceito de Ciência Aberta, que podemos definir como (Foster, 2017, para. 1):

Open Science is the practice of science in such a way that others can collaborate and contribute, where research data, lab notes and other research processes are freely available, under terms that enable reuse, redistribution and reproduction of the research and its underlying data and method.

A Ciência Aberta representa um desafio para a literacia da informação. A exigência formativa 
passa por articular a literacia da informação com a Ciência Aberta na base da formação e desenvolvimento de novas competências.

O objetivo da presente reflexão teórica é evidenciar os contributos da literacia da informação em contexto académico na dinâmica da produção de ciência e analisar os processos e as trajetórias a explorar no âmbito da Ciência Aberta. Por outras palavras, procura-se responder às seguintes questões: Quais são as áreas chave da Ciência Aberta que devem ser exploradas na literacia da informação? Quais são as novas competências a desenvolver pelos profissionais da informação de modo a articular a Ciência Aberta com a literacia da informação?

\section{A literacia da informação em contexto académico}

A complexificação das formas documentais e da sua organização, bem como o livre acesso à informação, levou a uma maior necessidade de formar para o seu bom uso. Assim, a literacia da informação surge como um conjunto de competências a desenvolver para a pesquisa, localização, seleção, avaliação e utilização da informação para uso pessoal, de forma ética e legal, particularmente no seio das bibliotecas. A Association of College \& Research Libraries [ACRL] refere que estas habilidades integradas "abrangem a descoberta reflexiva da informação, a compreensão de como a informação é produzida e valorizada, e o uso da informação na criação de novos conhecimentos e a participação de forma ética em comunidades de aprendizagem" (ACRL, 2015, p. 3).

Por seu turno, o contexto atual em que a informação é produzida e partilhada inclui novos formatos e novas plataformas de edição e disponibilização de documentos, designadamente no espaço digital. Neste novo ecossistema da informação todos os atores envolvidos, particularmente aqueles que trabalham com a informação escrita - investigadores, autores, editores, estudantes, professores, jornalistas, bibliotecários e cidadãos em geral, enquanto beneficiários da produção científica, técnica e literária -, sentem o impacto destas alterações (Lopes, Sanches, Andrade, Antunes, \& Alonso-Arévalo, 2016).

O ciclo de produção da informação está diretamente conectado com o papel das bibliotecas, particularmente as de ensino superior e de investigação. De facto, este ciclo pode explicar-se a partir de três etapas principais:

1. Inicia-se com uma pergunta de investigação que despoleta o processo de procura da informação em fontes primárias, bases de dados, bibliotecas, fomentando a produção intelectual.

2. Centra-se na escrita, na edição e na publicação, em que a reinterpretação de fontes e a recolha de dados fazem emergir novo conhecimento, que resulta em artigos científicos, capítulos, livros ou outros materiais.

3. Projeta-se e divulga-se a ciência produzida, procurando levar-se o conhecimento à sociedade através do acesso a bibliotecas, bases de dados, repositórios, motores de busca, entre outros. É a fase em que o conhecimento passa para a esfera pública, desejavelmente no âmbito da Ciência Aberta.

Recentemente, e ultrapassando o ciclo formal da publicação académica, impressa e física, conceitos como legitimidade e acessibilidade à informação digital suscitaram dúvidas na comunidade científica. Mas, gradualmente, os recursos da informação científica expandiram-se e diversificaram-se, tanto em volume como em formato, seguindo uma linha de garantia de controlo da qualidade do ciclo de produção da informação (Willinsky, 2006). O progressivo enfraquecimento dos filtros de qualidade da informação académica apela, assim, ao fortalecimento do pensamento crítico dos consumidores da informação científica (Basili, 2017). É legítima, por conseguinte, a transferência da responsabilidade dos conteúdos científicos das editoras para os consumidores, alertados e detentores de competências de análise e de avaliação. Investido de um conjunto de competências, o investigador fica habilitado a intervir na produção do conhecimento, passando a possuir a estrutura em que se apoiará a aprendizagem e abrindo a possibilidade ao potencial criativo; significa então que, mobilizando estas competências e porque as domina, em contextos de informação impressa ou digital, o investigador pode transformar essa mesma informação em novo conhecimento.

\section{Do acesso aberto à Ciência Aberta}

Assiste-se nos últimos trinta anos a processos de comunicação da ciência que se transformaram, adaptaram e evoluíram com a interatividade na comunicação de ciência. Mais recentemente, a expressão Ciência 2.0 rapidamente foi associada às redes sociais para investigadores, sistematizando mais uma das formas de comunicar ciência. Plataformas colaborativas de partilha, de edição de documentos, blogues, criação de perfis de investigador, o social tagging, o bookmarking, análises de fator de impacto, inclusive o impacto social na comunidade científica (o altmetrics), representam uma estrutura categorizada do ciclo de vida da investigação. O estudo de Nicholas e 
Rowlands (2011) refere que esta nova abordagem à ciência e à investigação permite identificar oportunidades de investigação, localizar novos parceiros, assegurar aporte científico, facilitar a revisão de literatura, a recolha e a análise de dados, a divulgação dos resultados, bem como a gestão do processo de investigação.

O princípio da Ciência Aberta tem sofrido alterações e adaptações à medida das políticas implementadas por cada país, dos avanços tecnológicos, mas também das pressões económicas e dos interesses públicos e privados. O acesso aberto aos dados da investigação está, por conseguinte, menos desenvolvido do que o acesso aberto às publicações científicas, facto que os stakeholders analisam de forma muito crítica, porque a Sociedade da Informação e a Sociedade do Conhecimento introduziram um conceito tecnológico e uma lógica na comunicação científica assente na abordagem económica. Nos tempos que correm, a Ciência Aberta sugere a livre circulação do conhecimento e a transparência do processo de investigação (Basili, 2017).

Em 2014, Ciência Aberta foi o termo escolhido pelos stakeholders durante a consulta pública para descrever as constantes mudanças ocorridas durante o processo de investigação, a colaboração dos investigadores, a partilha do conhecimento e a organização da ciência. Recorrendo ao uso da tecnologia digital, representa uma nova abordagem do processo científico baseada no trabalho colaborativo e em novas formas de disseminação do conhecimento. Na prática, a Ciência Aberta torna a ciência mais credível (integridade científica), mais fiável (transparência na confrontação de dados), mais eficiente (evita a duplicação de recursos) e mais eficaz face aos desafios societais, ajudando a encontrar respostas para os grandes problemas da atualidade (Boulton, 2013; European Commission, 2016a; RAND Europe, 2017; ver Antunes, 2016).

Segundo Open Science for the $21^{\text {st }}$ century: a declaration of all European academies, os dados científicos em acesso aberto são a chave para a construção de um novo edifício científico, podendo ser partilhados, utilizados em novas investigações e finalmente contribuir para uma nova dinâmica da descoberta científica (UNESCO, 2012). Também o Programa Horizonte 2020 refere que os dados resultantes de projetos de investigação financiada devem ser disponibilizados em acesso aberto (OpenAIRE, 2017).

Em 2015, Watson referiu que a Ciência Aberta era a forma de fazer investigação de forma completa, disponível e transparente, autorizando outros investigadores a construir mais conhecimento com base no conhecimento existente. A
Comissão Europeia (European Commission, $2016 b)$ vai mais longe. Sustentando os mesmos princípios, afirma que a Ciência Aberta envolve tecnologia e cultura em investigação num processo colaborativo e aberto. Também a OCDE (2015) assinala os benefícios da Ciência Aberta:

1. Na redução de custos da criação, transferência e reutilização de dados.

2. Na reutilização dos mesmos dados para mais ciência.

3. Na multiplicação de oportunidades para parcerias efetivas nas equipas de investigação.

4. No aumento da transparência e da qualidade no processo de validação.

5. Na maior rapidez de transferência do conhecimento.

6. Na promoção do envolvimento dos cidadãos na ciência.

Retoma-se a ideia, já explanada por Serrano Sanz, Holocher-Ertl, Kieslinger, García, e Silva (2014), de que através do conceito de Ciência Aberta se chega ao conceito de Ciência Cidadã: "A Ciência Cidadã refere-se ao compromisso do público em geral nas atividades de investigação científica quando os cidadãos contribuem ativamente para a ciência, seja através do seu esforço individual ou conhecimento circundante ou através das suas ferramentas e recursos" (p. 8). É interessante constatar como a ação individual, assente no entusiasmo pela ciência, e alavancada na Ciência Aberta, pode contribuir para a circulação e disseminação mais alargada do conhecimento científico (Boulton, 2013; Newman, et al., 2012).

A Ciência Aberta não sobrevive, porém, sem o acesso aberto - acesso aberto aos resultados da investigação, mas também aos dados da investigação e ao livre acesso à literatura científica. É inegável a lógica de edição/publicação, de comunicação académica e científica associada ao conceito de acesso aberto, que está, por sua vez, associada à literacia da informação (Suber, 2012). O trabalho de Basili (2017) apontou no mesmo sentido, ilustrando que, até agora, a literatura sobre a literacia da informação se tem concentrado muito mais no acesso aberto do que na Ciência Aberta. Mas todos os conteúdos associados ao acesso aberto - sejam as vias verde, dourada ou diamante, as licenças Creative Commons, a propriedade intelectual e os direitos de autor, as formas de comunicar ciência, as métricas de avaliação, entre outros - devem continuar a integrar as competências de literacia da informação a desenvolver em contexto académico. 
Vários estudos evidenciam que o acesso aberto beneficia a investigação e os investigadores e, na investigação financiada com recursos públicos, beneficia os contribuintes e aumenta o retorno do seu investimento na investigação (RAND Europe, 2017; Rodrigues, Swan, \& Baptista, 2013; Suber, 2012; Willinsky, 2005, 2006). Existem benefícios económicos bem como benefícios académicos e científicos:

- O acesso aberto amplifica o valor social da investigação.

- As políticas de acesso aberto amplificam o valor social das agências de financiamento e das instituições de investigação.

- Os custos do acesso aberto podem ser suportados sem adicionar mais dinheiro ao atual sistema de comunicação científica.

- O acesso aberto é consistente com as leis de direitos de autor em qualquer parte do mundo.

- O acesso aberto é consistente com os mais elevados padrões de qualidade.

O acesso aberto é, por conseguinte, somente uma das dimensões da Ciência Aberta, na medida em que o sistema científico é o campo de atuação desta: na disseminação do conhecimento (em acesso aberto), na criação de conhecimento (assegurando a transparência) e na transferência de conhecimento (disseminação) (Figura 1).

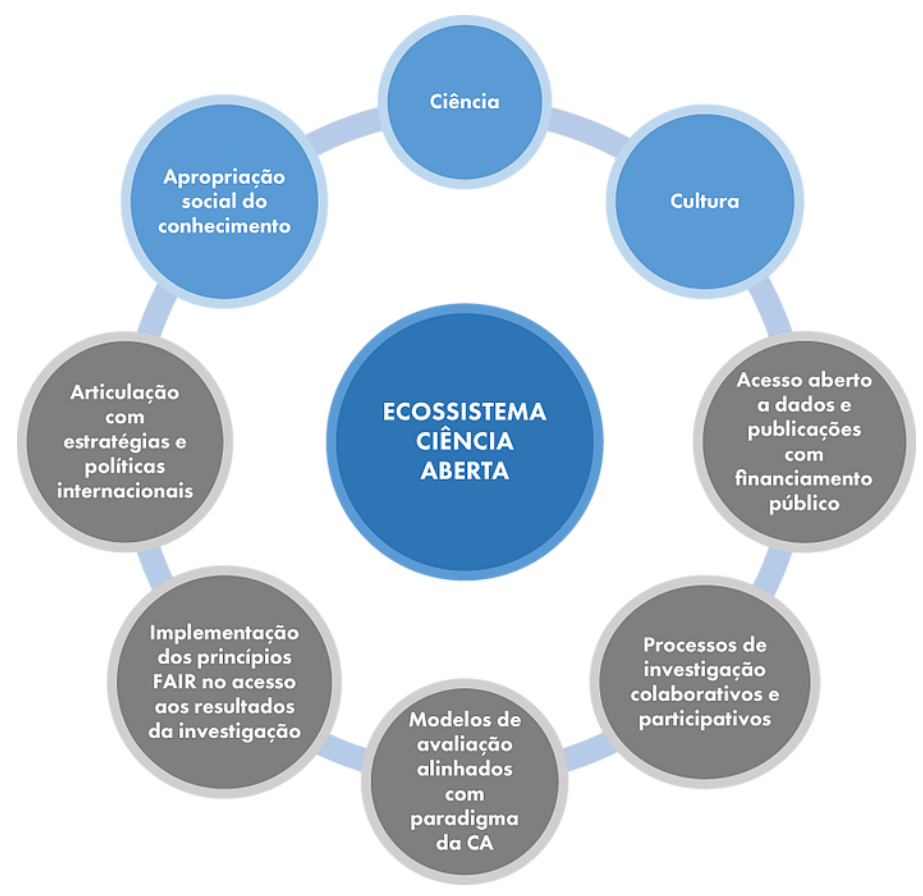

Figura 1. Ecossistema da Ciência Aberta (Fonte: Plataforma Ciência Aberta, http://www.ciencia-aberta.pt/sobre-ciencia-aberta)
A transferência do conhecimento produzido é o clímax do ciclo de investigação. Caraça (2012, p. $\mathrm{xv}$ ) refere mesmo que "apenas um caráter aberto garante a validade do conhecimento e permite uma avaliação real em relação à sua qualidade, isto é, sobre o valor da novidade criada através da sua circulação pela comunidade". Por outro lado, Shorley e Jubb (2013) debruçaram-se sobre a partilha de conhecimento científico que é possível ser feita com a comunidade académica e com a sociedade civil, sublinhando que a investigação científica não termina nesta etapa. De facto, a comunicação académica é uma parte vital do processo de investigação em si, inspirando outros investigadores por novos caminhos de descoberta e permitindo a criação de conexões entre os conceitos e os cidadãos.

\section{Políticas e desafios da Ciência Aberta em Portugal}

Em Portugal, os princípios orientadores da Ciência Aberta foram lançados em fevereiro de 2016 pela Secretaria de Estado da Ciência, Tecnologia e Ensino Superior (SECTES) e consubstanciados no documento Ciência Aberta | Conhecimento para todos (2016, p. 2):

Tornar a ciência mais aberta e acessível a todos, representando um desafio coletivo, fortalecerá a posição da ciência na sociedade, da mesma forma que contribuirá para uma sociedade mais qualificada e preparada para enfrentar o futuro. Ampliar a traslação do conhecimento científico para a sociedade e as empresas, tornando-o acessível à população de forma adequada, reforçará o impacto social da investigação e concorrerá para a sua valorização e reconhecimento.

A esta iniciativa seguiu-se a legislação de suporte sob a forma de Resolução do Conselho de Ministros $n^{\circ} 32 / 2016$, de 3 de junho de 2016. Este documento reconhece a importância do investimento no conhecimento, o qual constitui um pilar essencial do sucesso do desenvolvimento científico e tecnológico. Sublinha também que Portugal deve voltar a apostar no conhecimento como compromisso para o futuro, recuperando a confiança no seu sistema de ciência e tecnologia e no seu ensino superior, bem como garantir a sua aproximação e presença ativa na Europa da Ciência e do Conhecimento. Define, no ponto 1 , a criação de uma Agenda designada "Compromisso com o Conhecimento e a Ciência: o compromisso com o futuro» para o período 2016 a 2020. Entre outros objetivos, esta Agenda adota uma Política Nacional de Ciência Aberta, envolvendo o acesso aberto às publicações e aos dados científicos resultantes de investigação financiada por fundos públicos [ $\left.n^{\circ} 1, b\right)$, viii)], tendo em vista a promoção e a defesa de uma prática 
generalizada de Ciência Aberta e assumindo uma política científica comprometida com um paradigma de partilha do conhecimento e de aproximação da ciência à sociedade.
Após a publicação da legislação foram desenvolvidas várias iniciativas para a efetiva concretização da Ciência Aberta em Portugal (Tabela I):

\begin{tabular}{|c|c|}
\hline Iniciativas & Descrição dos conteúdos \\
\hline $\begin{array}{l}1^{\circ} \text { Fórum de Gestão de Dados de } \\
\text { Investigação } \\
\text { (23.09.2016) } \\
\text { (http://confdados.rcaap.pt/1forumgdi/) }\end{array}$ & $\begin{array}{l}\text { Dados abertos e repositórios de dados } \\
\text { Armazenamento de dados backup e preservação digital } \\
\text { Organização dos dados, documentação e metadados } \\
\text { Formação e desenvolvimento de competências } \\
\text { Os planos de gestão de dados }\end{array}$ \\
\hline $\begin{array}{l}\text { Criação da } \\
\text { Plataforma } \\
\text { Ciência Aberta } \\
\text { (outubro 2016) (http://www.ciencia- } \\
\text { aberta.pt/) }\end{array}$ & $\begin{array}{l}\text { Reúne informação, iniciativas e conteúdos formativos. Agrega a legislação aplicável } \\
\text { à Ciência Aberta, recursos eletrónicos, um glossário, MOOC (massive open online } \\
\text { courses) e projetos em curso. }\end{array}$ \\
\hline $\begin{array}{l}2^{\circ} \text { Fórum de Gestão de Dados de } \\
\text { Investigação } \\
\text { (31.03.2017) } \\
\text { (http://forumgdi.rcaap.pt/) }\end{array}$ & $\begin{array}{l}\text { Infraestruturas de investigação e preservação de dados } \\
\text { Proteção, licenciamento e reutilização de dados } \\
\text { Gestores de ciência no apoio à gestão de dados }\end{array}$ \\
\hline $\begin{array}{l}\text { Programa Ciência e Cultura } \\
\text { (março 2017) }\end{array}$ & $\begin{array}{l}\text { Seminários realizados: } \\
\text { Ciência e Cultura em Digital: como partilhar conhecimento - Discussão de estudos } \\
\text { de caso e de projetos de referência promovidos no setor da cultura. } \\
\text { Repositórios Digitais do Conhecimento - Constituição de um referencial de acesso } \\
\text { (Diretório de Repositórios Digitais) para o trabalho colaborativo e em rede. }\end{array}$ \\
\hline
\end{tabular}

Tabela I. Sinopse de algumas iniciativas da Ciência Aberta em Portugal

Em síntese, a Ciência Aberta envolve desde logo duas dimensões fundamentais: por um lado, a abertura e partilha dos resultados de investigação desde as publicações até aos dados da investigação; por outro lado, a abertura nos próprios métodos e ferramentas de investigação, tornando os processos abertos e colaborativos desde o seu início e procurando, quando adequado, o envolvimento de outros atores, para além da comunidade científica, na recolha e na análise dos dados de investigação, no que se designa de Ciência Cidadã. Maior abertura nos processos de investigação, maior rapidez na disseminação dos resultados em condições de poderem ser reutilizados, não apenas pela comunidade científica mas pela sociedade no seu conjunto; numa palavra, a Ciência Aberta resulta em vários benefícios e vantagens para a ciência e para a sociedade. Para a ciência, torna o seu progresso mais rápido e eficiente, como nos casos dos vírus Ébola e Zika que são assim enfrentados com os princípios da Ciência Aberta. E, simultaneamente, torna a ciência mais responsável, confiável e reprodutível, inibindo ou expondo erros, más práticas ou mesmo as fraudes científicas. Finalmente, para a sociedade, tornando a ciência mais responsiva aos desafios societais e facilitando a inovação e a apropriação de novo conhecimento para o desenvolvimento de novos produtos e serviços, ou seja, maximizando o retorno económico e social do investimento público na investigação e na ciência (Rodrigues, Swan, \& Baptista, 2013).

\section{Literacia da informação e Ciência Aberta: convergências}

Apresentando a ciência numa perspetiva sistémica, Basili (2017) caracteriza-a como um sistema organizado, cumulativo e estruturado de processos, objetos e ligações entre si, como um sistema aberto e não-isolado, propositadamente criado para produzir conhecimento, que depende da informação científica e preparado para partiIhar informação com a comunidade envolvente.

Retomando o objetivo da presente reflexão teórica, evidenciar os contributos da literacia da informação em contexto académico na dinâmica da produção de ciência e analisar os processos e as convergências a explorar no âmbito da Ciência Aberta, identificaram-se as seguintes trajetórias e áreas chave (Tabela II):

\begin{tabular}{ll}
\hline \multicolumn{1}{c}{ Las trayectorias de la Ciencia Abierta } \\
\hline $1 \quad \begin{array}{l}\text { Literacia da informação em contexto académico } \\
\text { como variável dependente do modus operandi do } \\
\text { processo científico }\end{array}$ \\
\hline $2 \quad \begin{array}{l}\text { Literacia da informação em contexto académico } \\
\text { como dimensão do processo de informação } \\
\text { científica }\end{array}$ \\
\hline $3 \quad \begin{array}{l}\text { Ciência como uma estrutura organizada e } \\
\text { complexa, com agentes e processos relacionados } \\
\text { entre si }\end{array}$ \\
\hline
\end{tabular}


4 Ciência, como um sistema não isolado, que implica canais diferenciados de circulação e de disseminação do conhecimento

5 Ciência, como um sistema não isolado, que implica a compreensão do papel dos diferentes stakeholders e os seus interesses na disseminação dos resultados da investigação

6 Informação académica e científica considerada sob qualquer formato, desde que explícito, registado e partilhado interna e externamente no seio da comunidade académica

7 Participação pública na ciência a considerar nas estratégias de literacia da informação em contexto académico

8 Redes sociais académicas que devem ser consideradas como novas formas de comunicação académica e científica

9 Interdisciplinaridade na ciência face ao princípio de sustentabilidade no financiamento da investigação (retorno socioeconómico)

10 Necessidade de compreensão das questões associadas à gestão e à curadoria de dados

Tabela II. Identificação das trajetórias da Ciência Aberta (construída a partir de Basili, 2017)

Neste contexto, a literacia da informação surge com novos e diferenciados tópicos: a) ciclo da comunicação científica; b) critérios de avaliação da fiabilidade da informação; e c) disseminação dos resultados da investigação.

Além das trajetórias enunciadas, a aprendizagem no ensino superior torna-se importante para esta reflexão, porque com a investigação, e no mesmo âmbito, promove a circulação de conhecimento.

Outro grande ponto de convergência deste estudo é a constatação de que a literacia da informação em contexto académico permanece em constante revisão e adaptação face à sua dependência das transformações vividas na Ciência Aberta, a qual requer, por si só, uma transformação cultural. Ambas, literacia da informação e Ciência Aberta, são interdependentes, particularmente no contexto do ensino superior, na medida em que a primeira capacita os indivíduos para as diversas fases do ciclo de produção científica e os resultados conseguidos pela Ciência Aberta maior acesso à informação - retroalimentam o ciclo de produção científica, só possível pela pesquisa, organização e divulgação do conhecimento produzido (Basili, 2017; Steinerová, 2016; Willinsky, 2005).

Como implicação prática, apresenta-se o exemplo do Programa FOSTER que criou um curso designado Integrating Open Science in

FAIR é um conjunto de princípios orientadores e critérios de qualidade para os dados de pesquisa: $\mathbf{F}$ ( findable $=$ pesquisáveis), $\mathbf{A}($ accessible $=$ acessíveis $), \mathbf{I}($ interoperable $=$ interoperáveis) e $\mathbf{R}$ (re-usable = reutilizáveis). information literacy education (FOSTER Programme, 2016), num formato online. O curso desenvolve as competências de literacia da informação a providenciar a doutorandos e investigadores de modo a garantir a salvaguarda da informação científica e dos dados de investigação (Tabela III).

\begin{tabular}{|c|c|}
\hline \multicolumn{2}{|c|}{ Competências } \\
\hline $\begin{array}{l}\text { Como } \\
\text { pesquisar? }\end{array}$ & $\begin{array}{l}\text { Em que se selecionam as fontes, se } \\
\text { definem search strings, se avalia a } \\
\text { informação, se criam alertas. Que } \\
\text { diferenças existem entre bases de dados e } \\
\text { plataformas científicas? Como construir } \\
\text { uma equação de pesquisa? O que são } \\
\text { operadores booleanos? }\end{array}$ \\
\hline $\begin{array}{l}\text { Como } \\
\text { organizar? }\end{array}$ & $\begin{array}{l}\text { Em que se gerem referências, se gerem } \\
\text { dados, se partilham dados e outra } \\
\text { informação. Porquê usar um software de } \\
\text { gestão de dados científicos? Quais são as } \\
\text { vantagens e desvantagens no } \\
\text { armazenamento dos dados científicos? O } \\
\text { que são princípios FAIR?1 Que software } \\
\text { usar? Que garantias oferece para a } \\
\text { segurança dos dados? Que critérios } \\
\text { considerar na escolha da rede de } \\
\text { colaboração e na partilha com outros } \\
\text { investigadores? Dever-se-á usar, ou não, } \\
\text { um fórum de discussão para a partilha de } \\
\text { experiências? Dever-se-ão partilhar, ou } \\
\text { não, os dados científicos depois da } \\
\text { investigação concluída? Existe, ou não, um } \\
\text { plano de gestão de dados? E porquê? }\end{array}$ \\
\hline $\begin{array}{l}\text { Como } \\
\text { divulgar? }\end{array}$ & $\begin{array}{l}\text { Em que se gere o impacto da produção } \\
\text { científica. O que saber antes de publicar? } \\
\text { Como escolher uma revista para publicar? } \\
\text { O que é o impacto científico? O que é a } \\
\text { open peer review? Como definir as co- } \\
\text { autorias num trabalho de investigação? } \\
\text { Que dados publicar e como publicá-los? } \\
\text { Como publicar em acesso aberto? O que } \\
\text { são as vias verde, dourada e diamante? }\end{array}$ \\
\hline
\end{tabular}

Tabela III. Programa FOSTER: integração da Ciência Aberta na formação em literacia da informação

Também como implicação prática, assinalam-se alguns estudos realizados em Portugal sobre a integração da literacia da informação no currículo académico (Andrade, 2016; Lopes, 2016; Lopes, \& Pinto, 2016) no âmbito do processo de ensinoaprendizagem. Os resultados revelam a importância da articulação da literacia da informação com a Ciência Aberta, na transformação da aprendizagem e do ensino através das tecnologias (recursos educativos abertos). Bibliotecas digitais e repositórios facilitam a reutilização de conteúdos digitais, permitindo o acesso a uma variedade de fontes e potenciando a formação de pensamento crítico e a criação de ideias mais complexas, ao mesmo tempo que incentivam os 
utilizadores a usar os recursos e a reinterpretálos (ver também o estudo de McGill, 2011). Os estudos assinalados realçam também a importância do bibliotecário integrado na promoção e no uso dos recursos eletrónicos na comunidade académica, centrado no seu papel de apoio ao difícil processo de usar informação e de a transformar em conhecimento. A este propósito, Revez (2014) menciona que as necessidades de formação e informação dos investigadores são difíceis de caracterizar, porém, a visibilidade parece ser um conceito-chave a considerar. E, sublinha (Revez, 2014, p. 163), "este tornar visível só é possível através de uma divulgação eficaz dos resultados da investigação, num meio em que o impacto deste conhecimento produzido é determinante para a captação futura de recursos, para o sucesso de lógicas de continuidade ao nível dos projetos e para o reconhecimento pela comunidade nacional e internacional da relevância de uma determinada academia".

Este contexto, em que a literacia e a investigação coabitam e contribuem para o desenvolvimento da Ciência Aberta e da aprendizagem foi largamente defendido e aprofundado por Basili (2017; ver também os contributos de Bruce, 2003, 2004), que propôs uma visão articulada em torno de três eixos: a literacia da informação, a formação em literacia da informação e a investigação. Nestes estudos, o principal argumento é o de que a literacia da informação é o somatório das diferentes formas de experienciar a informação; por isso, a formação em literacia da informação tem como objetivo ajudar os aprendentes (e também os investigadores) a alterar ou alargar o seu repertório de experiências face à informação.

Para a literacia da informação se consolidar como ferramenta formativa, capacitando os diferentes agentes do processo de investigação, responde-se à segunda questão: quais são as novas competências a desenvolver pelos profissionais da informação de modo a articular a Ciência Aberta com a literacia da informação?

De acordo com as Recomendações para as Bibliotecas do Ensino Superior de Portugal (GTBES, 2016), estes são os contributos da literacia da informação na Ciência Aberta para a capacitação dos profissionais de informação:

- Reafirmar a relevância das competências de literacia da informação na comunidade académica. Afirmar a necessidade de desenvolvimento da literacia da informação na comunidade académica, sublinhando, junto dos parceiros institucionais, a importância da creditação de competências de literacia da informação, incluindo nos curricula dos cursos iniciativas que promovam essas competências e garantindo que a biblioteca responde, de forma prospetiva e proativa, às solicitações dos docentes para apoio às atividades de ensino e investigação.

- Desenvolver competências dos profissionais das bibliotecas para apoio às atividades de ensino e aprendizagem. Apostar no reforço e desenvolvimento de competências técnicas e pedagógicas dos profissionais das bibliotecas e na criação de conteúdos online para as atividades de formação e transferência de conhecimento da Biblioteca, investindo no estabelecimento de parcerias com os docentes $\mathrm{e}$ na conceção e desenvolvimento de serviços de apoio ao ensino, concretizando iniciativas que promovam a partilha de conteúdos.

- Apoiar projetos editoriais de publicação académica e científica. Estar disponível para assumir novas funções no domínio da publicação e edição de revistas académicas e científicas, livros ou outras novas formas de partilhar ciência, apoiando os responsáveis na definição de políticas editoriais e modelos de negócio, bem como na coordenação editorial, através do desenvolvimento, manutenção e suporte de sistemas de informação que permitam o alojamento e a disseminação destas publicações.

- Assegurar repositórios institucionais alinhados com os padrões de interoperabilidade e preservação. Consolidar repositórios institucionais com as especificações técnicas que garantam os padrões de interoperabilidade e de preservação digital, reforçando o papel central do repositório nos ecossistemas institucionais e nacionais de informação académica e científica, nomeadamente na integração com os sistemas de gestão de ciência (CRIS - Current Research Information Systems) e na aplicação das políticas de Acesso Aberto.

- Criar serviços de apoio à gestão de dados científicos. Conceber serviços de apoio à gestão de dados científicos, alicerçados em planos de intervenção estratégica para atuação institucional que respondam às necessidades da gestão dos dados gerados e recolhidos na atividade dos investigadores, compreendendo o papel relevante das instituições de investigação no cumprimento das políticas de dados dos organismos financiadores da ciência e procurando a promoção de uma cultura de dados abertos que incentive a partilha, a identificação e a citação dos dados de investigação. 
- Potenciar o papel da biblioteca no apoio à investigação. Fomentar uma relação de confiança e parceria entre a biblioteca e os investigadores de forma a evidenciar as novas competências dos profissionais de informação no suporte à atividade de investigação, publicação e avaliação de desempenho, dotando os investigadores das ferramentas necessárias para aumentar a visibilidade do trabalho científico a nível individual e institucional, interpretar os indicadores de avaliação e aferir o impacto da produção científica.

- Fomentar parcerias com estruturas de apoio à comunidade académica. Desenvolver, de forma ativa e efetiva, parcerias com as estruturas e serviços de apoio aos estudantes, professores e investigadores, construindo soluções comuns, geradoras de mais-valias para a comunidade académica, potenciando serviços inovadores de caráter transversal.

Estas recomendações configuram-se como desafios para as instituições de ensino superior, afetando toda a comunidade e constituindo-se, para as bibliotecas e seus profissionais, como relevantes estímulos à implementação de novos serviços de apoio efetivo às atividades de aprendizagem e investigação, pautados por uma dimensão de pioneirismo tecnológico e aposta na colaboração e inovação.

\section{Conclusões}

No contexto em análise procurou-se demonstrar como é importante reconhecer as áreas chave em torno da Ciência Aberta para procurar assegurar que a literacia da informação corresponda, no apoio fornecido, aos objetivos daquela. Todos os envolvidos podem e devem compreender em que medida precisam de desenvolver maior perícia, particularmente se estão a liderar processos desta natureza.

Resulta desta análise que a exigência formativa em articular a literacia da informação com a Ciência Aberta, na base da formação e desenvolvimento de novas competências, deve considerar que:

- A Ciência Aberta permite a partilha do conhecimento entre a comunidade científica, a sociedade e as empresas, possibilitando desta forma ampliar o reconhecimento e o impacto social e económico da ciência.

- A Ciência Aberta é mais do que a disponibilização em acesso aberto de dados e publicações, é a abertura do processo científico enquanto um todo, reforçando o conceito de responsabilidade social científica.
- A implementação de uma prática de Ciência Aberta é também geradora de múltiplas oportunidades de inovação.

- Permite impulsionar o desenvolvimento de novos produtos, serviços, negócios e empresas.

A literacia da informação, em conclusão, assume-se como uma ferramenta de aprendizagem essencial para o desenvolvimento da Ciência Aberta, potenciando e capacitando o entendimento crítico dos conteúdos, a par do desenvolvimento e do progresso da investigação.

\section{Referencias}

Andrade, Isabel (2016). O curso de literacia da informação da NOVA Escola Doutoral: the road to information literacy. // Lopes, Carlos; Sanches, Tatiana; Andrade, Isabel; Antunes, Maria da Luz; \& Alonso-Arévalo, Julio (eds.). Literacia da informação em contexto universitário. Lisboa: Edições ISPA, 2016. 59-85.

Antunes, Maria da Luz (2016). Glossário da Ciência Aberta. Lisboa: Secretaria de Estado da Ciência, Tecnologia e Ensino Superior, 2016. http://www.ciencia-aberta.pt/glossario (2017-06-30).

Association of College \& Research Libraries [ACRL] (2015). Framework for information literacy for higher education. Chicago: American Library Association, 2015. www.ala.org/acrl/standards/ilframework (2017-02-15).

Basili, Carla (2017). Information literacy requirements for open science. // Sales, Dora; Pinto, María (eds.). Pathways into information literacy and communities of practice. Amsterdam: Chandos Publishing, 2017. 229-248.

Boulton, Geoffrey (2013). Reinventing open science for the $21^{\text {st }}$ century. // Rodrigues, Eloy; Swan, Alma; Baptista, Alice (eds.). Uma década de acesso aberto na UMinho e no mundo. Braga: Universidade do Minho, Serviços de Documentação, 2013. 239-250.

Bruce, Christine (2003). Seven faces of information literacy: towards inviting students into new experiences. Brisbane, AU: Queensland University of Technology. www.bestlibrary.org/digital/files/bruce.pdf (2017-02-15).

Bruce, Christine (2004). Information literacy as a catalyst for educational change: a background paper. // Danaher, Patrick Allan (ed.). Proceedings Lifelong Learning: whose responsibility and what is your contribution? The $3^{\text {rd }}$ International Lifelong Learning Conference. Yeppoon, Queensland, 2004.2 8-19. http://eprints.qut.edu.au/4977/1/4977_1.pdf (2017-0215).

Caraça, João (2012). Prefácio: para abrir a ciência. // Cardoso, Gustavo; Jacobetty, Pedro; Duarte, Alexandra (eds.). Para uma ciência aberta. Lisboa: Mundos Sociais, 2012. xiii-xv.

Cordón, Jose Antonio (2016). Prólogo. // Lopes, Carlos; Sanches, Tatiana; Andrade, Isabel; Antunes, Maria da Luz; \& Alonso-Arévalo, Julio (eds.). Literacia da informação em contexto universitário. Lisboa: Edições ISPA, 2016. xi-xiii.

European Commission (2016a). What is open science? FAQs on the open science policy platform. Brussels: European Commission, 2016. https://ec.europa.eu/research/openscience/index.cfm?pg=open-science-policy-platform-faqs (2017-01-03).

European Commission (2016b). The digital agenda for Europe: open science. Brussels: European Commission, 
2016. https://ec.europa.eu/digital-agenda/en/open-science (2017-02-23).

Facilitate Open Science Training For European Research [FOSTER] (2017). Definition: open science. Brussels: FOSTER, 2017. www. fosteropenscience.eu/foster-taxonomy/open-science-definition (2017-02-18).

FOSTER Programme. (2016). Integrating Open Science in information literacy education. Brussels: Author. Retrieved from https://www.fosteropenscience.eu/content/integrating-open-science-information-literacy-education

Grupo de Trabalho das Bibliotecas de Ensino Superior [GTBES] (2016). Recomendações para as bibliotecas de ensino superior. Lisboa: Associação Portuguesa de Bibliotecários, Arquivistas e Documentalistas, 2016. https://www.bad.pt/noticia/wp-content/uploads/2015/12/Recomendacoes_GTBES_2016_final.pdf (2017-07-02).

Lopes, Carlos (2016). As competências da literacia da informação integradas nos curricula académicos. // Lopes, Carlos; Sanches, Tatiana; Andrade, Isabel; Antunes, Maria da Luz; Alonso-Arévalo, Julio (eds.). Literacia da informação em contexto universitário. Lisboa: Edições ISPA, 2016. 87-102.

Lopes, Carlos; Pinto, María (2016). Autoavaliação das competências de informação em estudantes universitários. // Lopes, Carlos; Sanches, Tatiana; Andrade, Isabel; Antunes, Maria da Luz; \& Alonso-Arévalo, Julio (eds.). Literacia da informação em contexto universitário. Lisboa: Edições ISPA, 2016. 27-56.

Lopes, Carlos; Sanches, Tatiana; Andrade, Isabel; Antunes, Maria da Luz; Alonso-Arévalo, Julio (2016). Literacia da informação em contexto universitário. Lisboa: Edições ISPA, $2016.2 \mathrm{http}$ ://repositorio.ispa.pt/handle/10400.12/5067 (2017-06-30).

McGill, Lou (2011). Curriculum innovation: pragmatic approaches to transforming learning and teaching through technologies. London: JISC, 2011. www.jisc.ac.uk/media/documents/programmes/curriculumdelivery/curriculumdeliveryfinalreport.pdf (28 February 2017)

Newman, Greg; Wiggins, Andrea; Crall, Alycia; Graham, Eric; Newman, Sarah; Crowston, Kevin (2012). The future of citizen science: emerging technologies and shifting paradigms. // Frontiers in Ecology and the Environment. 10:6 (2012) 298-304.

Nicholas, David; Rowlands, Ian (2011). Social media use in the research workflow. // Information Services and Use. 31:1-2 (2011) 61-83.

OCDE (2015). Making open science a reality. Paris: OECD Publishing, 2015.

OpenAIRE (2017). How effective is Horizon 2020 so far. https://www.openaire.eu/how-effective-is-horizon2020so-far (2017-07-23).
Portugal. Resolução do Conselho de Ministros n 32/2016, de 3 de junho de 2016.

RAND Europe (2017). Open science monitoring: methodological note. Brussels: European Commission, 2017. https://ec.europa.eu/research/openscience/pdf/monitor/open_science_monitor_methodological_note.pdf (2017-03-01)

Revez, Jorge (2014). A formação de docentes e investigadores e as bibliotecas académicas: um desafio e uma proposta. // Cadernos BAD. 1 (2014) 155-171.

Rodrigues, Eloy; Swan, Alma; Baptista, Alice (eds.). Uma década de acesso aberto na UMinho e no mundo. Braga: Universidade do Minho, Serviços de Documentação, 2013

Secretaria de Estado da Ciência, Tecnologia e Ensino Superior [SECTES] (2016). Ciência Aberta | Conhecimento para todos. Lisboa: SECTES, 2016.

Serrano Sanz, Fermín; Holocher-Ertl, Teresa; Kieslinger, Barbara; Sanz García, Francisco; Silva, Cândida G. (2014). White paper on citizen science for Europe. Brussels: European Commission, 2014. www.zsi.at/object/project/2340/attach/White_Paper-Final-Print.pdf (2017-0223).

Shorley, Deborah; Jubb, Michael (2013). The future of scholarly communication. London: Facet, 2013.

Steinerová, Jela (2016). Open science and the research information literacy framework. // Kurbanoğlu, S.; Boustany, J.; Špiranec, S.; Grassian, E.; Mizrachi, D.; Roy, L. (eds.) Information literacy: key to an inclusive society $-4^{\text {th }} \mathrm{ECIL}$ 2016. London: Springer International Publishing. 277285. https://link.springer.com/chapter/10.1007/978-3319-52162-6_27 (2017-03-16).

Suber, Peter (2012). Open access. Massachusetts: MIT Press, 2012.

UNESCO (2012). Open Science for the $21^{\text {st }}$ century: declaration of ALL European Academies. http://www.unesco.org/new/fileadmin/MULTIMEDIA/HQ/Cl/Cl/pdf/news/ALLEA\%20Declaration\%20on\%200pen\%20Science.pdf (2017-06-30).

Watson, Mick (2015). When will 'open science' become simply 'science'? // Genome Biology. 16:1 (2015) 101

Willinsky, John (2005). The unacknowledged convergence of open source, open access, and open science. // First Monday. 10:8 (2005). http://www.firstmonday.org/ojs/index.php/fm/article/view/1265 (2017-07-09)

Willinsky, John (2006). The access principle: the case for Open Access to research and scholarship. Cambridge, MA: MIT Press, 2006

Enviado: 2017-04-01. Segunda versión: 2017-08-17. Aceptado: 2017-12-04. 
